\title{
Accuracy of 3D Range Scanners by Measurement of the Slanted Edge Modulation Transfer Function
}

\author{
Michael Goesele \\ Christian Fuchs \\ MPI Informatik \\ Saarbrücken, Germany \\ \{goesele,cfuchs,hpseidel\}@mpi-sb.mpg.de
}

\begin{abstract}
We estimate the accuracy of a $3 D$ range scanner in terms of its spatial frequency response. We determine a scanner's modulation transfer function (MTF) in order to measure its frequency response. A slanted edge is scanned from which we derive a superresolution edge profile. Its Fourier transform is compared to the Fourier transform of an ideal edge in order to determine the MTF of the device. This allows us to determine how well small details can be acquired by the $3 D$ scanner. We report the results of several measurements with two scanners under various conditions.
\end{abstract}

\section{Introduction}

Numerous 3D range scanning devices utilizing many different acquisition technologies have been built in the last decades by industrial as well as academic developers $[2,3,6,18]$. Comparing their performance in terms of accuracy is however difficult as each system has its specific strengths and weaknesses so that accuracy depends heavily on the test targets and measurement conditions used [6]. A system that can faithfully digitize a smooth surface of an object might not be able to acquire a sharp edge between two surfaces with sufficient accuracy. Beraldin et al. [1] used therefore multiple test targets to determine the accuracy the deviation from the exact shape - of range scanning systems for various conditions.

In contrast to previous work, we determine the accuracy of a range scanning system in terms of its spatial frequency response or its optical transfer function (OTF). The OTF determines the ability of the scanning system to reproduce different spatial frequencies present in an object. Sharp edges of an object to be scanned contain arbitrarily high spatial frequency components. A range scanning system which samples a finite number of surface points will never be able to reproduce a sharp edge faithfully unless it makes some assumptions about features present in the orig-

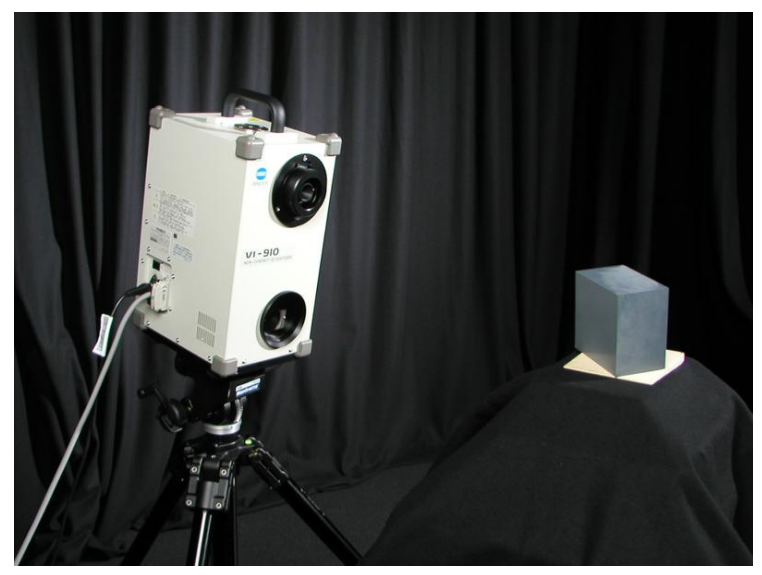

Figure 1. The setup used for test scans consisting of a Minolta VI-910 laser range scanner and the $90^{\circ}$ calibration target.

inal scene. Whether the reconstructed edge looks blurry or shows aliasing artifacts depends on the OTF of the scanning system resp. its modulation transfer function (MTF), the amplitude of the OTF.

Our work is inspired by the elegant and accurate slanted edge OTF measurement technique [17] for 2D imaging devices such as digital cameras or scanners and extends it into the third dimension. In principle, a single scan of a test target containing a straight sharp edge is sufficient to compute an estimate of the OTF. Once such a test target is available, a very simple acquisition setup such as depicted in Figure 1 is sufficient to perform this analysis.

This paper is organized as follows: after a review of previous work we explain our measurement principle in Section 3. We then discuss what an ideal MTF looks like (Section 4) and show the results of an empirical analysis of the proposed technique in Section 5. Section 6 gives several examples of MTFs measured under various conditions. We then conclude with an outlook on future work in Section 7. 


\section{Previous Work}

We first review some previous work on 3D range scanner accuracy and focus then on methods to determine the OTF of continuous and sampled systems.

\subsection{Range Scanner Accuracy}

Accuracy is commonly defined as the closeness of agreement between the result of a measurement and the value of the measurand [19]. Standard accuracy tests for 3D range scanners are often based on length measurement tests developed for coordinate measurement systems or for photogrammetry [2, 11]. Hebert and Krotkov [9] for example use multiple measurements of targets with known ground truth distance and compute the RMS error. Most commonly, accuracy is specified as range accuracy or as a multiple of the standard deviation $\sigma$ of a Gaussian fitted to the measurement results [7].

Chen et al. [6] point out that these accuracy values can only be compared if a standard measurement procedure is used including an exact specification of the test targets. The test targets should furthermore contain the same features such as curved surfaces or sharp edges that are encountered during the practical use of the scanner [1].

\subsection{OTF Measurement}

Characterizing imaging devices by their OTF is an established technique since many decades - an overview can be found for example in [21]. There are many techniques based on the observation of edge gradients, sine waves, square waves, or other features to determine the OTF of a continuous system. Using the Rayleigh criterion [4], the resolution of the system can be easily determined from the OTF [20]. Direct application of these techniques to sampled systems is however difficult due to noise, undersampling, and the anisotropic sampling performed by most sampled imaging systems [17].

Reichenbach et al. [17] introduced a knife-edge technique for OTF measurement of sampled imaging systems where a superresolution edge profile is computed from an image of a slanted edge. Multiple scan lines are registered to each other and combined into a single edge profile to overcome the difficulties due to undersampling and anisotropic sampling problems. Noise can be suppressed by smoothing or by more advanced filtering techniques [8]. The method is now part of several ISO standards for resolution measurements such as [10] but can also reveal many other characteristics of an imaging system [5, 20, 14].

Miles et al. [12] determined the OTF of an intensity image acquired by a ladar range imaging system. They did however not analyze the OTF of the associated range data.

In contrast to these approaches, we measure the modulation transfer function of a 3D scanning device using a single acquired range image. We use the MTF to derive an accuracy measure for range scanning systems that specifically takes the performance at object edges into account. Some work on this has already been reported by Paakkari and Moring [15] who included a set of 3D bar gratings into their performance tests of range imaging devices and carried out some limited experiments to determine the frequency response of a range imaging sensor.

\section{Measurement Principle}

The optical transfer function (OTF) describes the spatial response of a linear system. It is commonly used to describe the performance of an optical system [21]. The OTF consists of the modulation transfer function (MTF) encoding the change in amplitude and the phase transfer function (PTF) encoding the phase difference:

$$
\begin{aligned}
\operatorname{MTF}(s) & =|O(s) / I(s)| \\
\operatorname{PTF}(s) & =\phi(O(s))-\phi(I(s))
\end{aligned}
$$

where $I$ is an input sine wave with frequency $s$ and phase $\phi$, $O$ is the corresponding output sine wave with the same frequency but possibly different amplitude and phase. Using the principle of superposition for linear systems, an arbitrary input signal can be decomposed in its frequency components via Fourier transform, multiplied with the OTF, and transformed back using inverse Fourier transform to compute the system's response to the input signal.

\subsection{Slanted Edge Analysis for Three-Dimensional Range Scanning Systems}

The OTF of a sampled imaging system such as a digital camera can be computed using the knife-edge technique. While serving well for traditional analog systems, this technique has certain problems when dealing with sampled systems such as digital cameras. As one of these problems is undersampling, it is necessary to improve the spatial resolution of the device. The OTF can be measured beyond the Nyquist frequency of the device by placing an edge target slightly off-perpendicular to the scanning axes and averaging multiple scan lines (see Figure 2). Hereby a whole dimension is sacrificed to overcome the Nyquist limit.

We are using a slightly slanted edge of a cube instead of a flat knife-edge target in order to measure the OTF of a 3D scanner. A step edge function corresponding directly to a knife-edge target would be mathematically better to analyze the frequency response. It introduces however the problem of mixed pixels [9] which is of interest for long range scanners but not necessarily for object acquisition.

The target edge should be as sharp and straight as possible and the two surfaces adjacent to the edge should be diffuse reflecting and flat. The angle of the edge can also influence the measurements as shown in Section 5.2. 

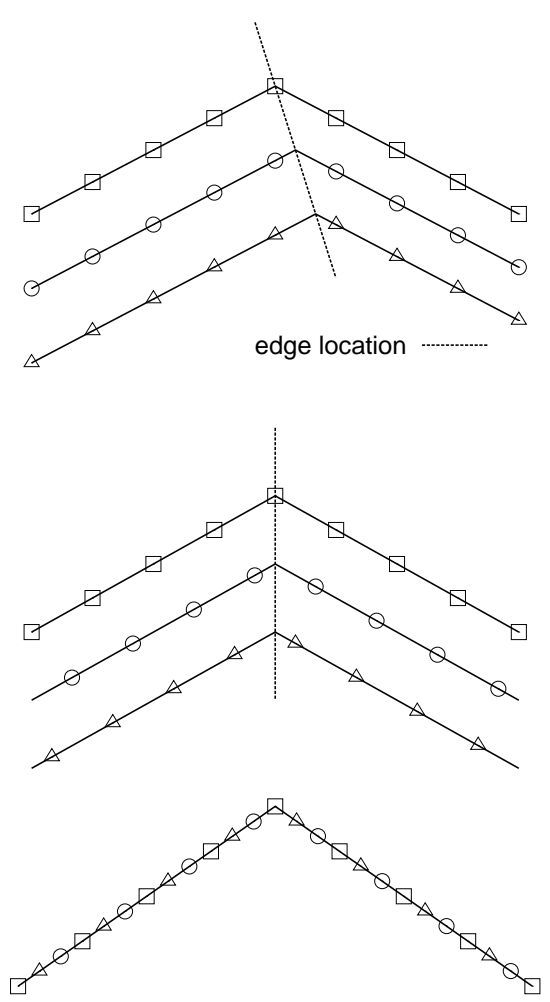

Figure 2. Registration and superposition of scanned points. After sampling the slanted edge with a regular sampling grid (top), the points are registered along the edge location (middle), and projected onto a plane to form a superresolution edge profile (bottom).

The larger the distance to the target is, the further away are the sample points from each other. Note that the Nyquist frequency can only be determined if the sample points are evenly spaced, i.e., the scan is not an arbitrary unstructured point cloud and if the scan shows a flat surface perpendicular to the scanning direction. If the scanning device returns points on a grid, it is however possible to compute the average distance between neighboring points in a certain region from which an estimate of the Nyquist frequency can be computed.

The focus of the device also affects the frequency response at the target edge. The edge should therefore be exactly in focus. If the scanner is equipped with an autofocus system, the focus setting should be verified because focusing exactly on a sharp edge can be difficult.

\subsection{Data Processing}

Our data processing starts once a single scan of a slanted edge has been acquired. The scan is saved as a range image retaining the individual scan lines. Next, it is automatically segmented by a histogram based technique using local normals. Two least-squares planes are fitted to the reliable data points on both sides of the edge which excludes the central region near the edge and noisy or incompletely sampled areas. The intersection of the planes is used to register the individual scan lines to one of the coordinate axes of the scanner's imaging sensor by a simple shearing operation. All data points are then projected onto a plane perpendicular to the registered line of intersection to form the superresolution edge profile (see Figure 2). Likewise, a "perfect" edge profile is computed from the sheared version of the fitted planes.

\subsection{Data Analysis}

The two generated profiles are then resampled perpendicular to the bisecting line of the perfect profile into $2^{n}$ bins centered around the peak of the profile using twofold oversampling compared to the estimated Nyquist frequency. Hereby some samples are discarded at both ends of the profiles. The ends of the binned profiles are moved to the horizontal axis and the profiles are componentwise multiplied with a modified Welch window [16]. Then each profile is rotated around one end point by $180^{\circ}$ in order to continue it periodically. The goal of these operations is that the only high frequency features of the profiles are the two peaks. Continuation errors around the zero line are suppressed. Figure 3 shows the two profiles.

Now the Fourier transform can be applied to each profile. If the whole process contains no numerical errors, the even Fourier coefficients should all be zero and we therefore neglect them when calculating the MTF estimate. The MTF estimate is then calculated by dividing the absolute value of the odd Fourier coefficients of the scanned profile by the corresponding Fourier coefficients of the perfect profile. Figure 5 shows plots of the Fourier coefficients and the calculated MTF estimate.

\section{The "Ideal" Modulation Transfer Function}

Before we continue by analyzing different MTF measurements, we first raise the question how an MTF plot can be interpreted and what the "ideal" MTF looks like.

For the case of a $2 \mathrm{D}$ imaging system such as a digital camera, Burns and Williams [5] separate the area of an MTF plot into several regions as given in Figure 4. The ideal MTF should fall completely inside a sweet spot starting at a unity modulation transfer for a constant signal. The modulus at the Nyquist frequency should be around 0.1 - the resolution limit given by the Rayleigh criterion $[4,20]$. If the MTF falls off too fast, high spatial frequencies are suppressed and the image looks blurry. An MTF that falls partially into the sharpening region above the sweet spot indicates that there is a danger of over sharpening, edge ringing, 


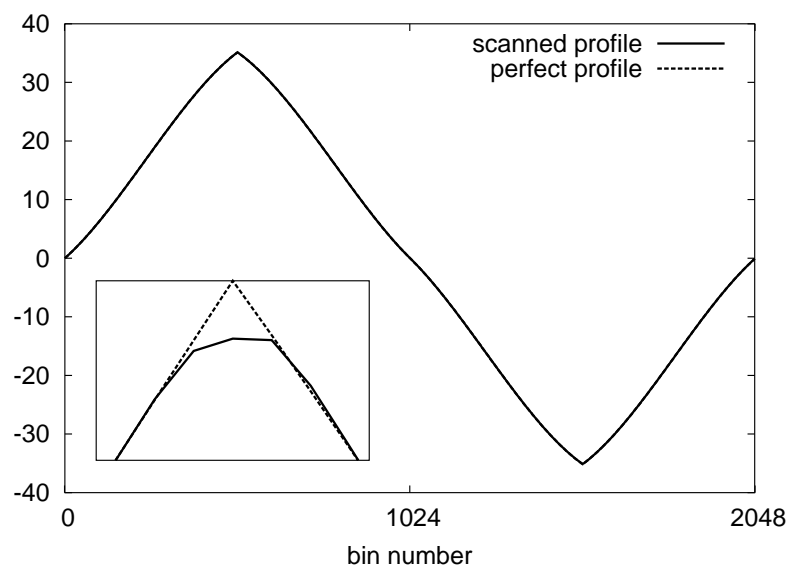

Figure 3. The superresolution and the "perfect" edge profile for a single scan. The insert shows a magnified view of the positive peak.

and increased noise. A significant amount of signal beyond the Nyquist frequency indicates undersampling of the scene leading to aliasing artifacts in the acquired images.

\subsection{Three-Dimensional Systems}

Whereas most images are viewed as captured or with minor post-processing applied, 3D range scans are often only the input data for a long chain of processing steps. Multiple scans are for example aligned and merged into a single point cloud from which eventually a three-dimensional model of the scanned object is generated. Each operation influences the overall MTF.

A generally desirable property is however that signals up to a certain frequency should be preserved, i.e., the MTF should be unity to ensure a faithful representation. Likewise, the MTF should be zero for high frequencies to suppress noise. Whether a steep falloff with the potential of ringing artifacts or a slow falloff that smoothly suppresses higher frequencies is better remains to be determined.

Merging multiple range scans can potentially increase the overall sampling rate. The merged scan can in that way sample a signal correctly even if it contains substantial frequency components beyond the Nyquist frequency of each individual scan. This approach is related to the way the superresolution edge is constructed for our MTF measurement technique.

\section{Empirical Analysis}

We tested our approach with a Minolta VI-910 laser triangulation scanner that acquires up to 307,000 points in a single scan by sweeping a laser plane through space and observing the reflected light. A $25 \mathrm{~mm}$ lens was mounted on

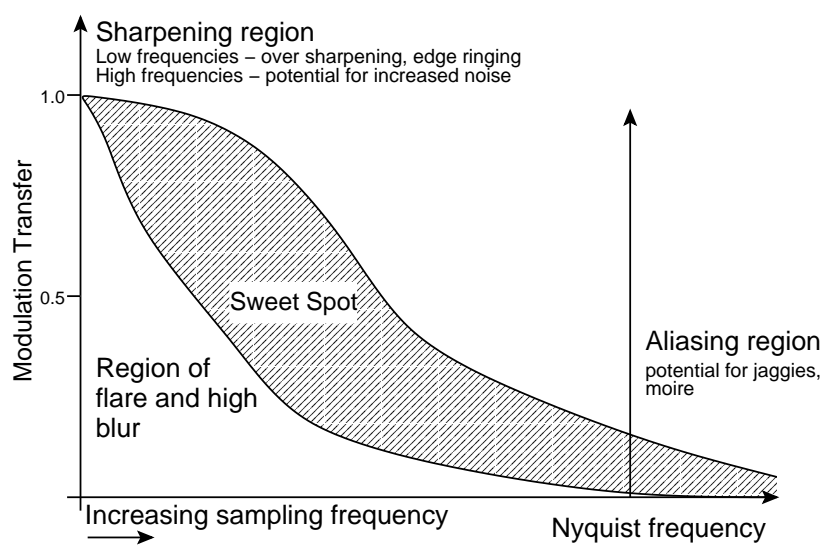

Figure 4. The ideal MTF for a 2D imaging system (adapted from Burns and Williams [5]). An MTF should ideally fall inside the "sweet spot". Different regions around it can be correlated with visible artifacts in the acquired images.

the scanner and no filtering was applied to the scans unless noted otherwise. More details about the scanner specification are available at the Minolta web site [13]. The scanner was controlled by Minolta's Polygon Editing Tool software.

Finding suitable test targets is difficult as they have to contain a sharp edge with a resolution larger than the measured MTFs. For our tests we used dark gray blocks of rigid $\mathrm{PVC}$ with precision milled edges of $80^{\circ}, 90^{\circ}$, and $100^{\circ}$ edge angle. The edge lengths varied between 75 and $108 \mathrm{~mm}$. The results below and in Section 6 suggest that the edges are of sufficient quality, however an independent test should be performed in the future.

The complete test setup for one configuration is depicted in Figure 1.

\subsection{Analysis of a Single Scan}

The profiles in Figure 3 were computed from a single range scan of the $90^{\circ}$ target consisting of 159,780 surface points to which the noise filter of the Polygon Editing Tool was applied (see Section 6.2). After binning the data into 2048 bins and applying the Fourier transform, even and odd Fourier coefficients are plotted in separate graphs (see Figure 5). The horizontal axis is labeled in multiples of the sampling resolution. Note that the even coefficients of the perfect edge are several orders of magnitude smaller than the odd coefficients. Furthermore, the even coefficients of the scanned edge stay on a constant noise level which is also reached by the odd coefficients around 0.9 times the sampling resolution.

The MTF plot is quite noisy which could be improved by smoothing it, averaging multiple plots, or by filtering the 

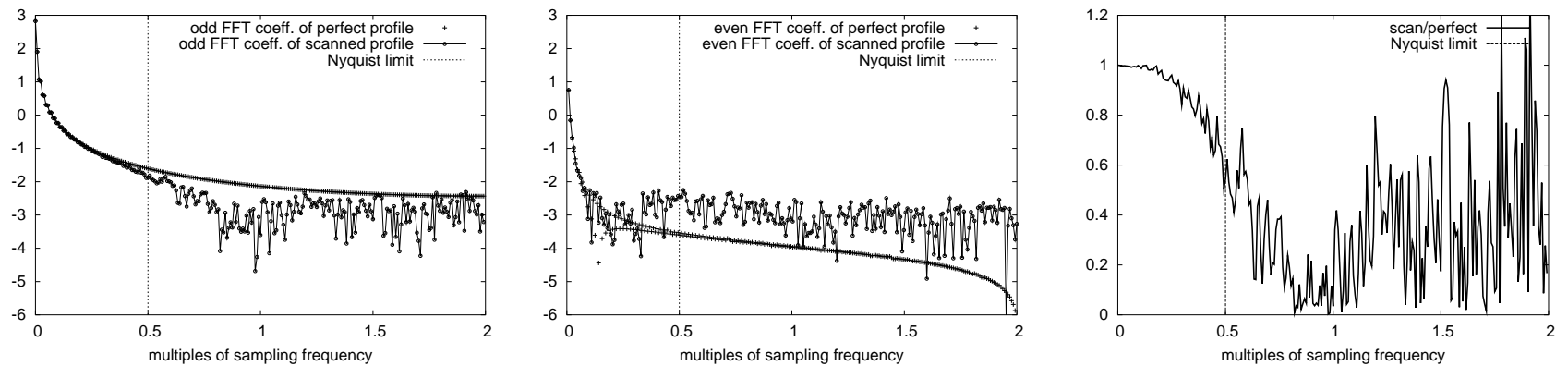

Figure 5. Data computed from the profiles in Figure 3. Odd (left) and even (middle) coefficients of the FFT on a logarithmic scale. Note the different orders of magnitude. Right: MTF plot for the odd coefficients. The scale of the horizontal axis is derived from the estimated Nyquist frequency and shows the twofold supersampling.

input data such as in [8]. Nevertheless, the general shape of the curve is clearly visible and shows for example a quite substantial contribution beyond the estimated Nyquist limit which is a potential source of aliasing. Furthermore, the plot has a clear minimum between 0.8 and 1.0 caused by the smoothing effect of the noise removal (see Section 6.2 for a detailed comparison). The signal beyond the sampling frequency seems to be caused by remaining noise in the input data which is confirmed by the results of the repeatability test in Section 5.2.

\subsection{Repeatability and Robustness}

As a repeatability test, we performed 20 scans of the $90^{\circ}$ target with constant scanner settings where we slightly varied the position and orientation of the test target. Figure 6 shows a plot of the average MTF computed from these scans and the standard deviation per coefficient. While the technique gets unreliable for high frequencies beyond twice the Nyquist limit, it shows that the MTF can be recovered quite robustly for frequencies up to this point.

To evaluate how much the results depend on the edge angle of the test target, we performed a test series with the $80^{\circ}, 90^{\circ}$, and $100^{\circ}$ test targets and the VI-910 with no filtering applied. Each target was scanned 3 times and analyzed (see Figure 7). The averaged MTF plots show that the general shape is largely independent of the edge angle. Higher frequencies are however suppressed if the target angle gets smaller. In principle, this could be a quality problem of the target. We presume however that the main problem lies in the fact that sharper angled edges are harder to digitize. It is therefore likely that smoothing errors occur at higher frequencies. Although this behavior violates the linear system assumption (there is no unique $\operatorname{MTF}(s)$ for a given frequency $s$ ), we still believe that our analysis is applicable and can help to understand the behavior of a scanning device.

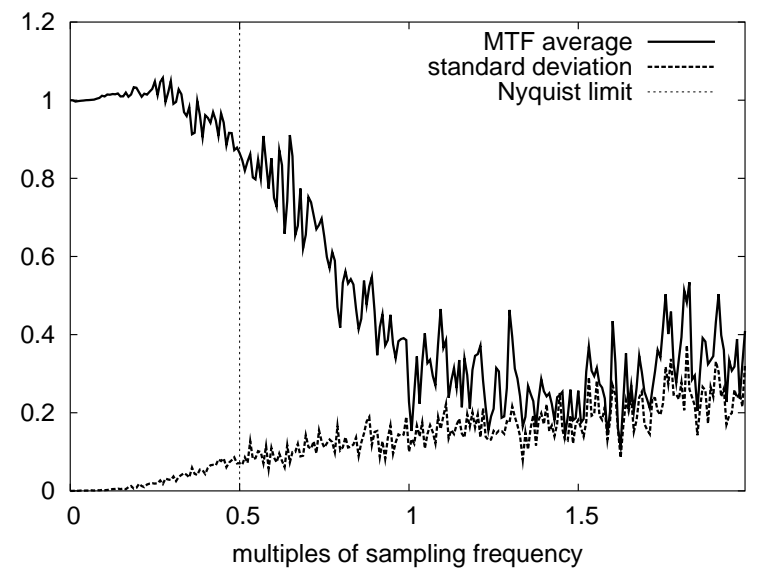

Figure 6. Average MTF for 20 scans and standard deviation per frequency.

\section{Further Examples}

We show in this section several effects that can be observed by analyzing the MTF of a 3D scanner. We first compare the MTFs computed for horizontal and vertical edges, show then the effects of different filtering operations, and finally compare the MTFs of two different scanner models.

\subsection{Horizontal and Vertical MTF}

The scanning principle of sweeping a laser plane and observing the reflections on the objects is a highly anisotropic process. This can lead to asymmetries in the MTFs for horizontal and vertical edge directions. This phenomenon can for example be observed for flatbed image scanners where a linear sensor is moved across the scan [20]. It is therefore advisable to at least compute MTFs for both directions using horizontal and vertical edges [17]. 


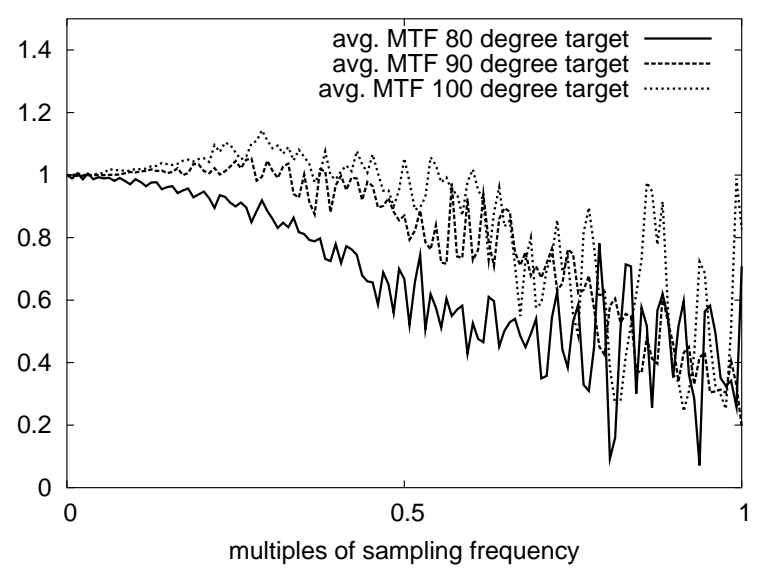

Figure 7. Average MTF for 3 scans and different test targets with edge angles of $80^{\circ}, 90^{\circ}$, and $100^{\circ}$.

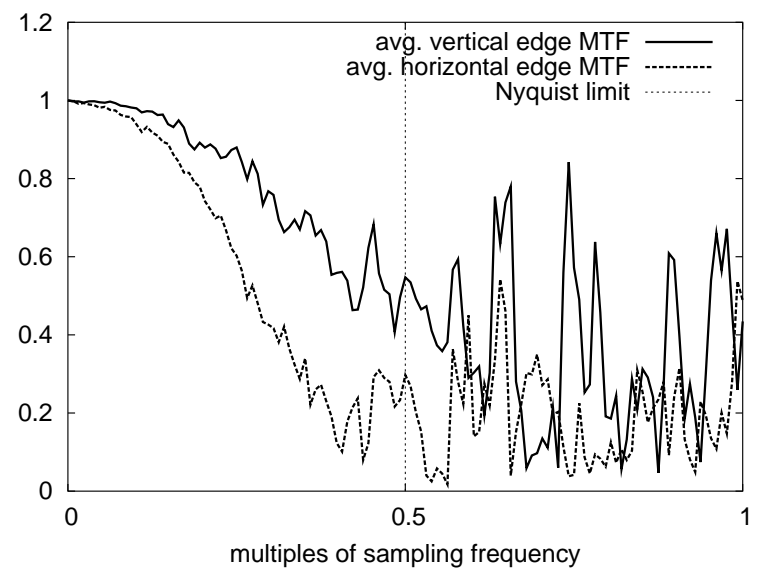

Figure 8. Comparison between the MTFs for a horizontal and a vertical edge.

The asymmetry of the MTF is confirmed by the plots in Figure 8; the falloff is steeper for the horizontal edge MTF. This can be caused by the fact that the vertical edge MTF is mainly determined by the resolution of the imaging sensor while the horizontal edge MTF depends as well on several other factors such as the number of positions of the laser plane or the width of the laser line. Furthermore, laser position detection can be more difficult when the projected laser line is almost parallel to the edge.

\subsection{Scan Processing}

The Minolta Polygon Editing Tool allows the application of several filters to a captured 3D scan after the acquisition but before exporting the data: noise filtering, high quality, and a combination of the two. The effects of this filtering
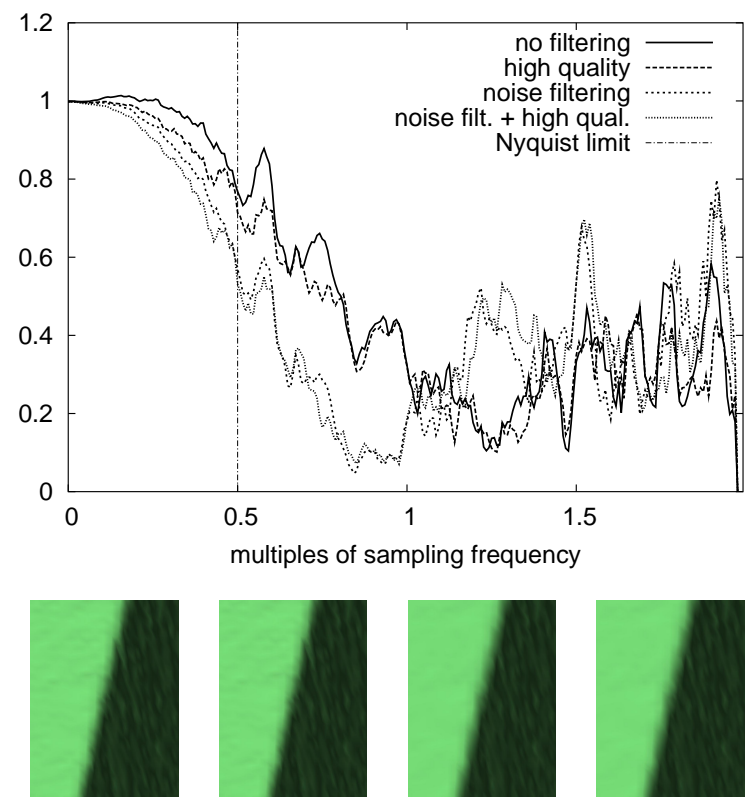

Figure 9. Influence of export filters on the MTF. No filter, the high quality filter, the noise filter, and the noise + high quality filter were applied to the same scan. The bottom row shows shaded scans of parts of the edge where some smoothing effect of the noise filter is visible (from left to right: no filter, high quality, noise, high quality + noise)

on the scan and on the computed MTFs are shown in Figure 9. It can be clearly seen that the noise filter suppresses a frequency band around and beyond the Nyquist frequency whereas the high quality filter has a rather small influence on the MTF. The combination of both filters has almost no additional effect on the MTF compared to the noise filter alone.

After exporting, the Polygon Editing Tool also offers the possibility to smooth the scanned object. We applied this filter $0,1,5$, and 10 times with a weight of 0.5 to the same scan used above. The resulting MTFs are shown in Figure 10. It can be seen that the MTF drops much earlier if more smoothing is applied. The images of the smoothed edges show less aliasing than the unfiltered version.

\subsection{Scanner Comparison}

When comparing MTFs of different scanners with different resolutions and consequently also different Nyquist limits, either a relative or an absolute comparison can be performed. For a relative comparison, the MTFs have to be normalized to the Nyquist frequency. Then the plots allow a comparison of how well the scanners behave within their 

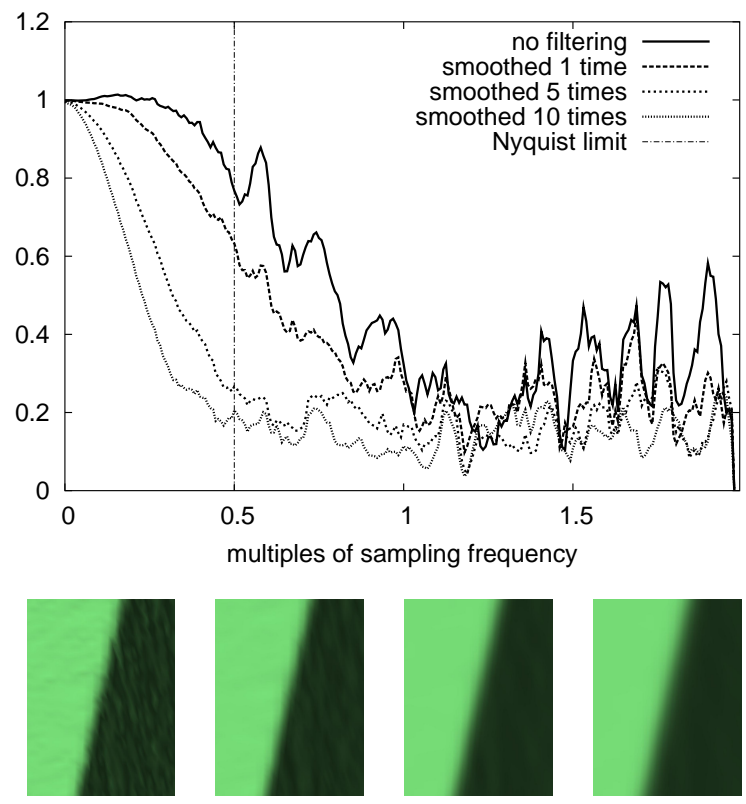

Figure 10. Influence of smoothing. The smoothing operation of the Polygon Editing Tool is $0,1,5$, and 10 times applied to the unfiltered edge scan from Figure 9 with parameter setting 0.5 . The corresponding renderings of parts of the edge. The effect of the smoothing operation is clearly visible.

respective theoretical limits. For an absolute comparison, the MTFs have to be normalized to the same resolution so that the behavior for same scale details can be compared.

We included a Minolta VI-700 - an earlier model than the Minolta VI-910 with lower resolution - in the test. Table 1 gives the details of two analyzed test scans whose MTFs are presented in Figure 11. The plot shows that the response of the VI-700 is quite flat up to the Nyquist limit and then drops quickly which means that the scanner should not suffer much from aliasing. Some frequencies are however exaggerated where the MTF is above unity. Due to its higher Nyquist limit, the VI-910 can sample higher frequencies.

\section{Conclusion and Future Work}

In this paper, we have shown, that the modulation transfer function of a 3D range scanner can be robustly determined using a slanted edge technique. Although dealing with three-dimensional data poses some additional problems compared to the MTF measurements for 2D imaging devices, the general simplicity of the slanted edge technique prevails. A single scan of a target provides sufficient data for the MTF calculation.

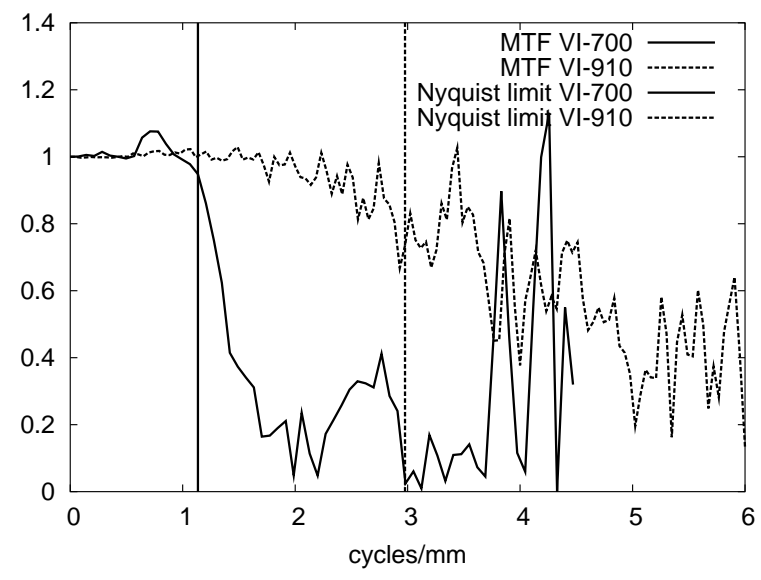

Figure 11. An MTF computed for the VI-700 scanner. Note the differences compared to the VI-910. The horizontal axes have been rescaled to allow for an absolute comparison of the two scanners (see Section 6.3).

\begin{tabular}{|l|c|c|}
\hline & VI-910 & VI-700 \\
\hline \hline $\begin{array}{l}\text { number of points used for } \\
\text { superresolution }\end{array}$ & 159,778 & 19,336 \\
\hline $\begin{array}{l}\text { number of bins used for su- } \\
\text { perresolution }\end{array}$ & 512 & 128 \\
\hline $\begin{array}{l}\text { average distance between } \\
\text { sampled points in mm }\end{array}$ & 0.167972 & 0.440337 \\
\hline
\end{tabular}

Table 1. Values used for computing the MTF estimates shown in Figure 11

There remain some open questions for future work. These include the best choice of material and surface finish for the test targets as well as performing test series with different target materials. Another question is - as already raised in Section 4 - to determine what the "ideal" MTF should look like and how an MTF should therefore be interpreted for 3D scanners. This will strongly depend on further processing steps applied to the scan. Furthermore, we would like to compare multiple scanners based on different scanning principles.

\section{Acknowledgements}

We would like to thank Minolta Europe GmbH for providing test scanners for this project. This work was funded in part by the DFG Schwerpunktprogramm V3D2 and by the European Community within the scope of the ViHAP3D Project IST-2001-32641 "Virtual Heritage: High-Quality 3D Acquisition and Presentation." 


\section{References}

[1] J.-A. Beraldin, S. F. El-Hakim, and F. Blais. Performance evaluation of three active vision systems built at the national research council of canada. In Proceedings of the Conference on Optical 3-D Measurements Techniques, pages 352361, 1995.

[2] F. Bernardini and H. Rushmeier. The 3D model acquisition pipeline. Computer Graphics Forum, 21(2):149-172, 2002.

[3] F. Blais. A review of 20 years of range sensor development. In Videometrics VII, Proceedings of SPIE-IS\&T Electronic Imaging, volume 5013, pages 62-76, 2003.

[4] M. Born and E. Wolf. Principles of optics: electromagnetic theory of propagation, interference and diffraction of light. Cambridge University Press, 7. (expanded) edition, 1999.

[5] P. D. Burns and D. Williams. Image resolution and MTF for digital cameras and scanners. In Tutorial Notes, IS\&T PICS Conference 2001, 2001.

[6] F. Chen, G. M. Brown, and M. Song. Overview of threedimensional shape measurement using optical methods. $O p$ tical Engineering, 39(1):10-22, January 2000.

[7] G. Dalton. Reverse engineering using laser metrology. Sensor Review, 18(2):92-96, 1998.

[8] T. A. Fischer and J. Holm. Electronic still picture camera spatial frequency response measurement. In Proceedings of IS\&T's 47th Annual Conference/ICPS, pages 626-630, 1994.

[9] M. Hebert and E. Krotkov. 3D measurements from imaging laser radars: how good are they? Intl. Journal of Image and Vision Computing, 10(3):170-178, April 1992.

[10] ISO 12233:2000: Photography - electronic still-picture cameras - resolution measurements, 2000.

[11] C. P. Keferstein and M. Marxer. Testing bench for laser triangulation sensors. Sensor Review, 18(3):183-187, 1998.

[12] B. Miles, J. Land, A. Hoffman, W. Humbert, B. Smith, A. Howard, J. Cox, M. Foster, D. Onuffer, S. Thompson, T. Ramrath, C. Harris, and P. Freedman. Field testing protocols for evaluation of 3D imaging focal plane array ladar systems. In Laser Radar Technology and Applications VII, Proceedings of SPIE, volume 4723, pages 43-56, 2002.

[13] Minolta web site, www.minolta-3d.com.

[14] Y. Okano. Influence of image enhancement processing on SFR of digital cameras. In Proceedings of IS\&T's PICS Conference 1998, pages 74-78, 1998.

[15] J. Paakkari and I. Moring. Method for evaluating the performance of range imaging devices. In Industrial Applications of Optical Inspection, Metrology and Sensing, Proceedings of SPIE, volume 1821, pages 350-356, 1993.

[16] W. H. Press, S. A. Teukolsky, W. T. Vetterling, and B. P. Flannery. Numerical Recipes in C: The Art of Scientific Computing. Cambridge Univ. Press, 2nd edition, 1994.

[17] S. E. Reichenbach, S. K. Park, and R. Narayanswamy. Characterizing digital image acquisition devices. Optical Engineering, 30(2):170-177, February 1991.

[18] R. Scopigno, C. Andujar, M. Goesele, and H. P. A. Lensch. 3D data acquisition. In Tutorial Notes, Eurographics Conference 2002, 2002.
[19] B. N. Taylor and C. E. Kuyatt. Guidelines for evaluation and expressing the uncertainty of NIST measurement results. Technical Report NIST 1297, National Institute of Standards and Technology (NIST), 1994.

[20] D. Williams and P. D. Burns. Diagnostics for digital capture using MTF. In Proceedings of IS\&T's PICS Conference 2001, pages 227-232, 2001.

[21] T. L. Williams. The Optical Transfer Function of Imaging Systems. Institute of Physics Publishing, London, 1999. 